\title{
Aspects of string phenomenology in particle physics and cos- mology
}

\author{
I. Antoniadis ${ }^{1,2, a}$ \\ ${ }^{1}$ LPTHE, UMR CNRS 7589 Sorbonne Universités, UPMC Paris 6, 75005 Paris, France \\ ${ }^{2}$ Albert Einstein Center, Institute for Theoretical Physics, Bern University, Sidlerstrasse 5, CH-3012 Bern
}

\begin{abstract}
I discuss possible connections between several scales in particle physics and cosmology, such the the electroweak, inflation, dark energy and Planck scales. In particular, I discuss the physics of extra dimensions and low scale gravity that are motivated from the problem of mass hierarchy, providing an alternative to low energy supersymmetry. I describe their realization in type I string theory with D-branes and I present the main experimental predictions in particle accelerators and their implications in cosmology. I also show that low-mass-scale string compactifications, with a generic D-brane configuration that realizes the Standard Model by open strings, can explain the relatively broad peak in the diphoton invariant mass spectrum at $750 \mathrm{GeV}$ recently reported by the ATLAS and CMS collaborations.
\end{abstract}

\section{Introduction}

If String Theory is a fundamental theory of Nature and not just a tool for studying systems with strongly coupled dynamics, it should be able to describe at the same time particle physics and cosmology, which are phenomena that involve very different scales from the microscopic four-dimensional (4d) quantum gravity length of $10^{-33} \mathrm{~cm}$ to large macroscopic distances of the size of the observable Universe $\sim 10^{28} \mathrm{~cm}$ spanned a region of about 60 orders of magnitude. In particular, besides the $4 \mathrm{~d}$ Planck mass, there are three very different scales with very different physics corresponding to the electroweak, dark energy and inflation. These scales might be related via the scale of the underlying fundamental theory, such as string theory, or they might be independent in the sense that their origin could be based on different and independent dynamics. An example of the former constrained and more predictive possibility is provided by $\mathrm{TeV}$ strings with a fundamental scale at low energies due for instance to large extra dimensions transverse to a four-dimensional braneworld forming our Universe [1]. In this case, the $4 \mathrm{~d}$ Planck mass is emergent from the fundamental string scale and inflation should also happen around the same scale [2].

The appropriate framework of low string scale is type I string theory with D-branes. Gauge and gravitational interactions have different origin. The latter are described by closed strings, while the former emerge as excitations of open strings with endpoints confined on D-branes [3]. This leads to a braneworld description of our universe, which should be localized on a hypersurface, i.e. a membrane extended in $p$ spatial dimensions, called $p$-brane (see Fig. 1). Closed strings propagate in all nine

ae-mail: ignatios.antoniadis@upmc.fr 
dimensions of string theory: in those extended along the $p$-brane, called parallel, as well as in the transverse ones. On the contrary, open strings are attached on the $p$-brane. Obviously, our $p$-brane

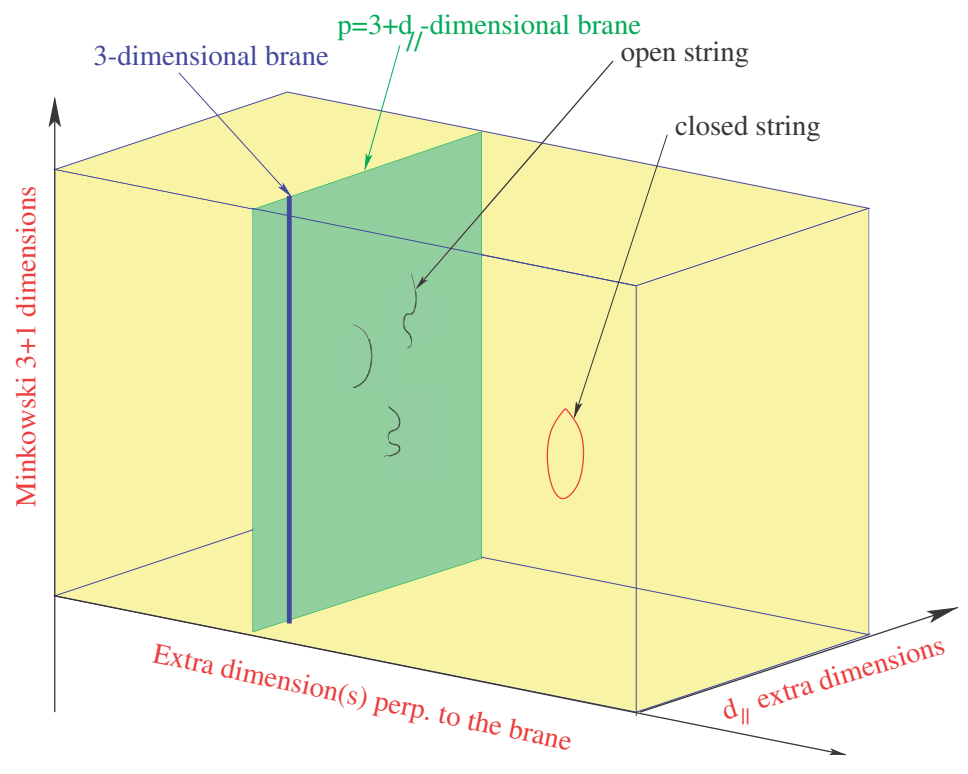

Figure 1. In the type I string framework, our Universe contains, besides the three known spatial dimensions (denoted by a single blue line), some extra dimensions $\left(d_{\|}=p-3\right)$ parallel to our world $p$-brane (green plane) where endpoints of open strings are confined, as well as some transverse dimensions (yellow space) where only gravity described by closed strings can propagate.

world must have at least the three known dimensions of space. But it may contain more: the extra $d_{\|}=p-3$ parallel dimensions must have a finite size, in order to be unobservable at present energies, and can be as large as $\mathrm{TeV}^{-1} \sim 10^{-18} \mathrm{~m}$ [4]. On the other hand, transverse dimensions interact with us only gravitationally and experimental bounds are much weaker: their size should be less than about $0.1 \mathrm{~mm}[5]$.

\section{Framework of low scale strings}

In type I string theory, the different origin of gauge and gravitational interactions implies that the relation between the Planck and string scales is not linear as in the heterotic string. The requirement that string theory should be weakly coupled, constrain the size of all parallel dimensions to be of order of the string length, while transverse dimensions remain unrestricted. Assuming an isotropic transverse space of $n=9-p$ compact dimensions of common radius $R_{\perp}$, one finds:

$$
M_{P}^{2}=\frac{1}{g^{4}} M_{s}^{2+n} R_{\perp}^{n}, \quad g_{s} \simeq g^{2} .
$$

where $g_{s}$ is the string coupling. It follows that the type I string scale can be chosen hierarchically smaller than the Planck mass at the expense of introducing extra large transverse dimensions felt only 
by gravity, while keeping the string coupling small [1]. The weakness of $4 \mathrm{~d}$ gravity compared to gauge interactions (ratio $M_{W} / M_{P}$ ) is then attributed to the largeness of the transverse space $R_{\perp}$ compared to the string length $l_{s}=M_{s}^{-1}$.

An important property of these models is that gravity becomes effectively $(4+n)$-dimensional with a strength comparable to those of gauge interactions at the string scale. The first relation of Eq. (1) can be understood as a consequence of the $(4+n)$-dimensional Gauss law for gravity, with

$$
M_{*}^{(4+n)}=M_{s}^{2+n} / g^{4}
$$

the effective scale of gravity in $4+n$ dimensions. Taking $M_{s} \simeq 1 \mathrm{TeV}$, one finds a size for the extra dimensions $R_{\perp}$ varying from $10^{8} \mathrm{~km}, .1 \mathrm{~mm}$, down to a Fermi for $n=1,2$, or 6 large dimensions, respectively. This shows that while $n=1$ is excluded, $n \geq 2$ is allowed by present experimental bounds on gravitational forces $[5,6]$. Thus, in these models, gravity appears to us very weak at macroscopic scales because its intensity is spread in the "hidden" extra dimensions. At distances shorter than $R_{\perp}$, it should deviate from Newton's law, which may be possible to explore in laboratory experiments (see Fig. 2).

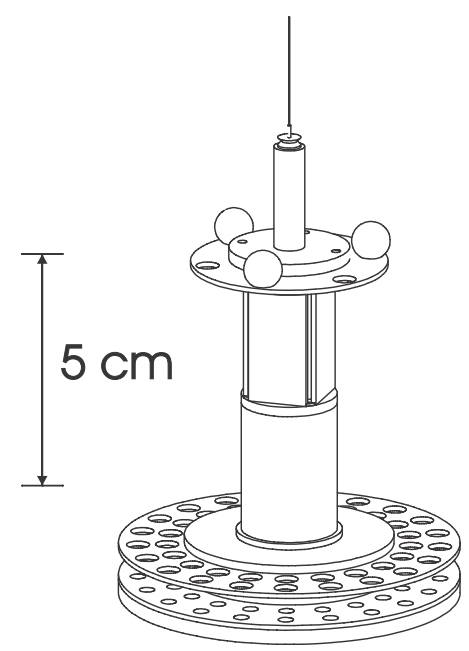

Figure 2. Torsion pendulum that tested Newton's law at $55 \mu \mathrm{m}$.

\subsection{Experimental implications in accelerators}

We now turn to the experimental predictions of TeV scale strings. Their main implications in particle accelerators are of four types, in correspondence with the four different sectors that are generally present:

1. New compactified parallel dimensions; In this case $R M_{s} \gtrsim 1$, and the associated compactification scale $R_{\|}^{-1}$ would be the first scale of new physics that should be found increasing the beam energy $[4,7]$. The main consequence is the existence of KK excitations for all Standard Model particles that propagate along the extra parallel dimensions. These can be produced on-shell at LHC as new resonances [8] (see Fig. 3). 


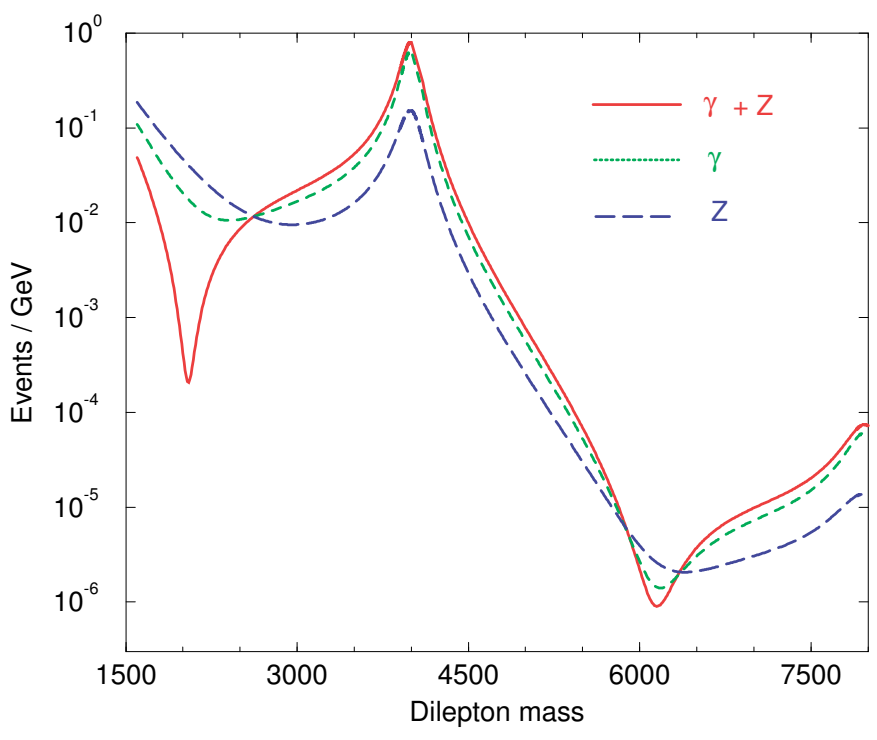

Figure 3. Production of the first KK modes of the photon and of the $Z$ boson at LHC, decaying to electronpositron pairs. The number of expected events is plotted as a function of the energy of the pair in $\mathrm{GeV}$.

2. New extra large transverse dimensions and low scale quantum gravity,. The main experimental signal is gravitational radiation in the bulk from any physical process on the world-brane [9].

3. Genuine string and quantum gravity effects. Direct production of string resonances in hadron colliders leads generically to a universal deviation from Standard Model in jet distribution [10]. In particular, the first Regge excitation of the gluon has spin 2 and a width an order of magnitude lower than the string scale, leading to a characteristic peak in dijet production; similarly, the first excitations of quarks have spin 3/2. The dijet (left) and $\gamma+$ jet (right) cross-sections are shown in Fig. 4 for LHC energies, while Fig 5 shows the Signal-to-Noise ratio of the lowest massive Regge excitations for a $100 \mathrm{TeV}$ future hadron collider [11].

4. Extra $U(1)$ 's arising generically in D-brane models as part of unitary gauge group factors. They obtain in general masses due to four- or higher-dimensional anomalies, via the so-called GreenSchwarz anomaly cancellation mechanism involving axionic fields from the closed string sector. The resulting masses are therefore suppressed by a loop factor compared to the string scale. From the low energy point of view, they gauge global symmetries of the Standard Model (SM), such as the baryon and lepton number. An important property of the anomaly cancellation mechanism is that the anomalous $U(1)$ gauge bosons acquire masses leaving behind the corresponding global symmetries unbroken in perturbation theory. Thus, this is a way to guarantee proton stability (from unbroken baryon number) and avoid large Majorana neutrino masses (from unbroken lepton number) due to dimension-5 operators involving two higgses and two leptons that are suppressed only by the TeV string scale. Such extra $U(1)$ s have interesting properties and distinct experimental signatures [12-15]. 

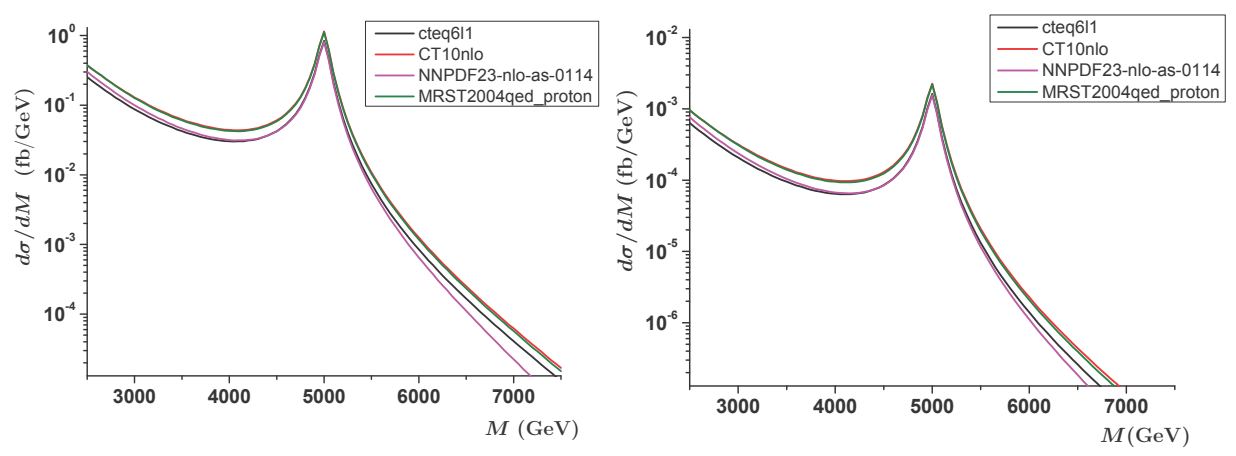

Figure 4. Production of the first Regge excitations at LHC in the dijet (left) and $\gamma+$ jet (right) channels, for $M_{s}=5 \mathrm{TeV}$. The cross-section is plotted as a function of the invariant mass $M$.
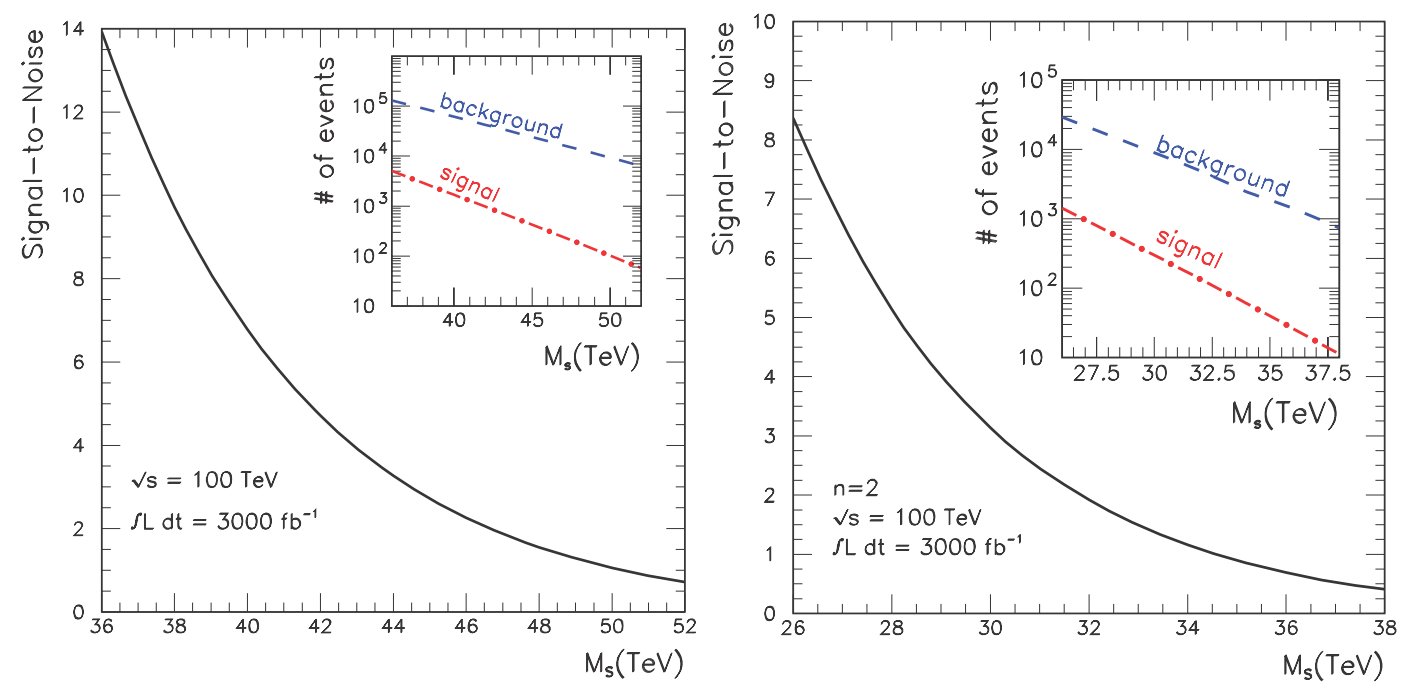

Figure 5. Dijet signal-to-noise ratio of the lowest $n=1$ (left) and $n=2$ (right) Regge excitations for a future $100 \mathrm{TeV}$ hadron collider.

5. Concerning possible micro-black hole production, note that a string size black hole has a horizon radius $r_{H} \sim 1$ in string units, while the Newton's constant behaves as $G_{N} \sim g_{s}^{2}$. It follows that the mass of a $d$-dimensional black hole is [16]: $M_{\mathrm{BH}} \sim r_{H}^{d / 2-1} / G_{N} \simeq 1 / g_{s}^{2}$. Using the value of the SM gauge couplings $g_{s} \simeq g^{2} \sim 0.1$, one finds that the energy threshold $M_{\mathrm{BH}}$ of microblack hole production is about four orders of magnitude higher than the string scale, implying that one would produce $10^{4}$ string states before reaching $M_{\mathrm{BH}}$.

On the other hand, there exist interesting implications in non accelerator table-top experiments due to the exchange of gravitons or other possible states living in the bulk. 


\section{$3750 \mathrm{GeV}$ diphotons from closed string states}

One can show that low-mass-scale string compactifications, with a generic D-brane configuration that realizes the standard model by open strings, can explain the peak in the diphoton invariant mass spectrum at $750 \mathrm{GeV}$ recently reported by the ATLAS and CMS collaborations.

Indeed, the ATLAS [17] and CMS [18] collaborations reported excesses of events over expectations from standard model (SM) processes in the diphoton mass distribution around $750 \mathrm{GeV}$, using (respectively) $3.2 \mathrm{fb}^{-1}$ and $3.3 \mathrm{fb}^{-1}$ of data recorded at a center-of-mass energy $\sqrt{s}=13 \mathrm{TeV}$, targeting spin-0 and spin- 2 resonances. This could be interpreted as decays of a new massive particle $\varphi$. The ATLAS Collaboration, reported for spin- 0 the largest deviation from the background-only hypothesis for $M_{\varphi} \sim 750 \mathrm{GeV}$ and $\Gamma_{\text {total }} \approx 45 \mathrm{GeV}$. The local significance iq $3.9 \sigma$, while the global significance is around $2 \sigma$. For the spin-2 resonance, both the local and global significances are somewhat smaller: $3.6 \sigma$ and $1.8 \sigma$, respectively. The CMS analysis gives the largest excess for $M_{\varphi}=760 \mathrm{GeV}$ and $\Gamma_{\text {total }} \approx 11 \mathrm{GeV}$ and has a local significance of $2.8 \sigma$ for spin- 0 and $2.9 \sigma$ spin-2 hypothesis. After taking into account the effect of searching for several signal hypotheses, the significance of the excess is reduced to $<1 \sigma$. The CMS Collaboration also reported a combined search on data collected at $\sqrt{s}=13 \mathrm{TeV}$ and $\sqrt{s}=8 \mathrm{TeV}$. For the combined analysis, the largest excess is observed at $M_{\varphi}=750 \mathrm{GeV}$ and $\Gamma_{\text {total }}=0.1 \mathrm{GeV}$. The local significance is $\approx 3.4 \sigma$ and the global significance $1.6 \sigma$.

Fitting the LHC13 data with a resonance yields a cross section times branching ratio of

$$
\sigma_{\mathrm{LHC} 13}(p p \rightarrow \varphi+\text { anything }) \times \mathcal{B}(\varphi \rightarrow \gamma \gamma) \approx\left\{\begin{array}{cl}
(10 \pm 3) \mathrm{fb} & \text { ATLAS } \\
(6 \pm 3) \mathrm{fb} & \text { CMS }
\end{array},\right.
$$

at $1 \sigma$ [19]. On the other hand, no diphoton resonances were seen in the data at $\sqrt{s}=8 \mathrm{TeV}$, although both ATLAS [20] and CMS [21] data show a mild upward fluctuation at invariant mass of $750 \mathrm{GeV}$. The lack of an excess at $\sqrt{s}=8 \mathrm{TeV}$ allows a quite precise limit to be placed on the corresponding cross section at $\sqrt{s}=13 \mathrm{TeV}$. The most stringent limit comes from the CMS search $\sigma_{\mathrm{LHC} 8}(p p \rightarrow$ $\varphi+$ anything $) \times \mathcal{B}(\varphi \rightarrow \gamma \gamma)<2.00 \mathrm{fb}$ at $95 \%$ CL [21]. This implies that if the diphoton cross section grows by less than about a factor of 3 or 3.5 the LHC 8 data are incompatible with the LHC13 data at 95\% CL.

More recently, we proposed a model [22] to explain the data in which the resonance production mechanism is calculable in string based dynamics, with large extra dimensions [1]. In our proposal the observed diphoton excess originates from a closed string excitation $\varphi$ living on the compact space of generic intersecting D-brane models that realize the SM chiral matter contents and gauge symmetry $[23,24]$. Namely we consider extensions of the SM based on D-brane string compactifications with large extra dimensions [1]. The basic unit of gauge invariance for D-brane constructions is a $U(1)$ field, so that a stack of $N$ identical D-branes eventually generates a $U(N)$ theory with the associated $U(N)$ gauge group; for $N=2$, the gauge group can be $S p(1) \cong S U(2)$ rather than $U(2)$. In the presence of many D-brane types, the gauge group becomes a product form $\prod U\left(N_{i}\right)$, where $N_{i}$ reflects the number of D-branes in each stack. In the perturbative regime, gauge interactions emerge as excitations of open strings ending on D-branes, with gauge bosons due to strings attached to stacks of D-branes and chiral matter due to strings stretching between intersecting D-branes.

Our explanation of the peak in the diphoton invariant mass spectrum is independent of the structure of the D-brane model. Nevertheless, to motivate the discussion we adopt a minimal model containing 4 stacks of D-branes. The basic setting of the gauge theory is given by $U(3)_{C} \times S p(1)_{L} \times U(1)_{L} \times$ $U(1)_{I_{R}}[15,25,26]$. In the bosonic sector the open strings terminating on the QCD stack contain, in addition to the $S U(3)_{C}$ octet of gluons $g_{\mu}^{a}$, an extra $U(1)$ boson $C_{\mu}$, most simply the manifestation 


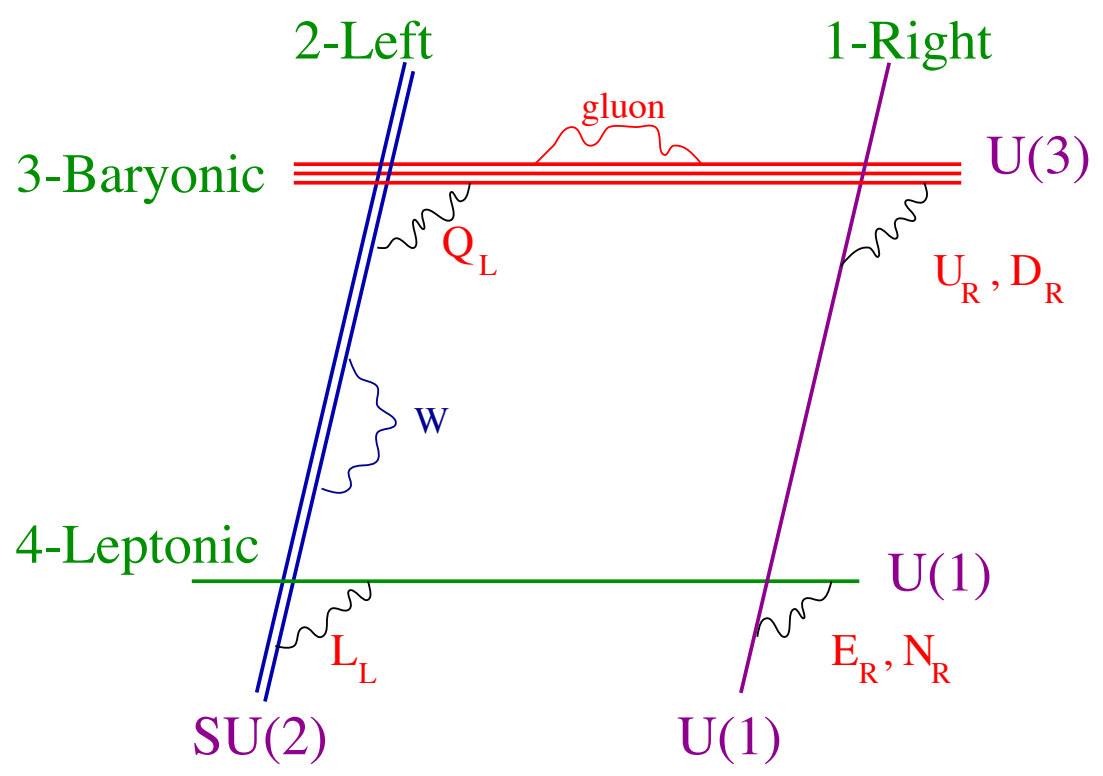

Figure 6. Pictorial representation of the $U(3)_{B} \times S U(2)_{L} \times U(1)_{L} \times U(1)_{I_{R}}$ D-brane model.

Table 1. Chiral spectrum of SM fields in the 4 stack D-brane model. We have added the right handed neutrino stretching between the lepton brane and the right brane.

\begin{tabular}{clccrrr}
\hline \hline Fields & Sector & Representation & $Q_{B}$ & $Q_{L}$ & $Q_{I_{R}}$ & $Q_{Y}$ \\
\hline$U_{R}$ & $3 \leftrightharpoons 1^{*}$ & $(3,1)$ & 1 & 0 & 1 & $\frac{2}{3}$ \\
$D_{R}$ & $3 \leftrightharpoons 1$ & $(3,1)$ & 1 & 0 & -1 & $-\frac{1}{3}$ \\
$L_{L}$ & $4 \leftrightharpoons 2$ & $(1,2)$ & 0 & 1 & 0 & $-\frac{1}{2}$ \\
$E_{R}$ & $4 \leftrightharpoons 1$ & $(1,1)$ & 0 & 1 & -1 & -1 \\
$Q_{L}$ & $3 \leftrightharpoons 2$ & $(3,2)$ & 1 & 0 & 0 & $\frac{1}{6}$ \\
$N_{R}$ & $4 \leftrightharpoons 1^{*}$ & $(1,1)$ & 0 & 1 & 1 & 0 \\
$H$ & $2 \leftrightharpoons 1$ & $(1,2)$ & 0 & 0 & 1 & $\frac{1}{2}$ \\
\hline \hline
\end{tabular}

of a gauged baryon number. The $S p(1)_{L} \simeq S U(2)$ stack is a terminus for the electroweak gauge bosons $W_{\mu}^{a}$. The $U(1)_{Y}$ boson $Y_{\mu}$ that gauges the usual electroweak hypercharge symmetry is a linear combination of $C_{\mu}$, and the $U(1)$ bosons $B_{\mu}$ and $X_{\mu}$ terminating on the separate $U(1)_{L}$ and $U(1)_{I_{R}}$ branes. A schematic representation of the D-brane construct is shown in Fig. 6. The general properties of the chiral spectrum are summarized in Table 1.

One can check by inspection that the hypercharge,

$$
Q_{Y}=\frac{1}{2} Q_{I_{R}}+\frac{1}{6} Q_{B}-\frac{1}{2} Q_{L},
$$


is anomaly free. However, the $Q_{B}$ (gauged baryon number) is not anomaly free and we expect this anomaly to be canceled via a Green-Schwarz mechanism involving the exchange of twisted RamondRamond (RR) closed string states [27-31]. There is an explicit mass term in the Lagrangian for the new gauge field $-\frac{1}{2} M^{\prime 2} Y_{\mu}^{\prime} Y^{\prime \mu}$ whose scale comes from the compactification scheme. The scalar that gets eaten up to give the longitudinal polarization of the $Y^{\prime}$ is a closed string field and there is no extra Higgs particle [32]. In addition to the intermediate RR field, which is absorbed by the $Y^{\prime}$ in the anomaly cancellation, there is a closed string mode $\varphi$ which couples to the anomaly free combination of the hypercharge (4). It can be either a scalar field from the Neveu-Schwarz sector that is complexified with the RR state absorbed by $Y^{\prime}$, or another RR pseudo-scalar (axion) coupled to $F \tilde{F}$.

There are two properties of the scalar $\varphi$ that are necessary for explaining the $750 \mathrm{GeV}$ signal. It should be a special closed string state with dilaton-like or axion-like coupling to $F^{2}$ (respectively to $F \tilde{F}$ ) of the electromagnetic field, but may be decoupled from $G^{2}$ of color $S U(3)$. The couplings of closed string states to gauge fields do indeed distinguish between different D-brane stacks, depending on the localization properties of D-branes with respect to $\varphi$ in the compact dimensions. More specifically, it is quite natural to assume that $\varphi$ is a closed string mode that is associated to the wrapped cycles of the (lepton) $U(1)_{L}$ and (right isospin) $U(1)_{I_{R}}$ stack of D-branes, however is not or only weakly attached to the wrapped cycle of (left) $S p(1)_{L}$ or the color $S U(3)$ stack of D-branes. In this way, we may avoid unwanted dijet signals. Actually, within a selection of string based explanations of the resonance [33-42] our proposal is uniquely exemplified by the possible suppression of dijet topologies in the final state. ${ }^{1}$ By choice, we may also allow a coupling $\varphi$ to $G^{2}$. This is possible by modifying the localization properties of D-branes with respect to $\varphi$ in the internal space.

Since the string mass scale is now known to be larger than $M_{s} \approx 7 \mathrm{TeV}$ [49], the mass $M_{\varphi} \approx$ $750 \mathrm{GeV}$ must be suppressed with respect to the string scale by some anomalous loop corrections. Because $\varphi$ is a twisted closed string localized at an orbifold singularity, its coupling to $\gamma \gamma$ should be suppressed by $M_{s}^{-1}$, provided the bulk is large [14]. With this in mind, we parametrize the coupling of $\varphi$ to the photon by the following vertex

$$
\frac{c_{\gamma \gamma}}{M_{s}} \varphi F^{2}
$$

To remain in the perturbative range, we also require $c_{\gamma \gamma} \lesssim 2 \pi$. The partial decay width of $\varphi$ to diphotons then follows as

$$
\Gamma_{\gamma \gamma}=\frac{c_{\gamma \gamma}^{2}}{4 \pi} \frac{M_{\varphi}^{3}}{M_{s}^{2}} .
$$

Let us first assume the diphoton signal is produced via photon-photon fusion with $\varphi$ as the resonance state. Following [50], herein we include the elastic-elastic processes (already considered in [22]) as well as elastic-inelastic and inelastic-inelastic contributions. The elastic production is suppressed with respect to inelastic by about an order of magnitude. However, elastic photoproduction events result in forward and backward protons which can be detected by forward detectors installed by ATLAS [51] and CMS [52]. Therefore, the detection of two unbroken protons in the final state, with $M_{p p}$ paired to $M_{\gamma \gamma}$, may be a promising way to reduce the background in the future [53,54].

The total photo-production cross section at LHC13 is [50]

$$
\sigma_{\mathrm{LHC} 13}(\gamma \gamma \rightarrow \varphi \rightarrow \gamma \gamma)=4.1 \mathrm{pb}\left(\frac{\Gamma_{\text {total }}}{45 \mathrm{GeV}}\right) \mathcal{B}^{2}(\varphi \rightarrow \gamma \gamma)
$$

\footnotetext{
${ }^{1}$ A related stringy explanation in which $\varphi$ can be produced through photon fusion has been put forward in [43]. Alternative axion and dilaton models have been discussed in [44-48].
} 


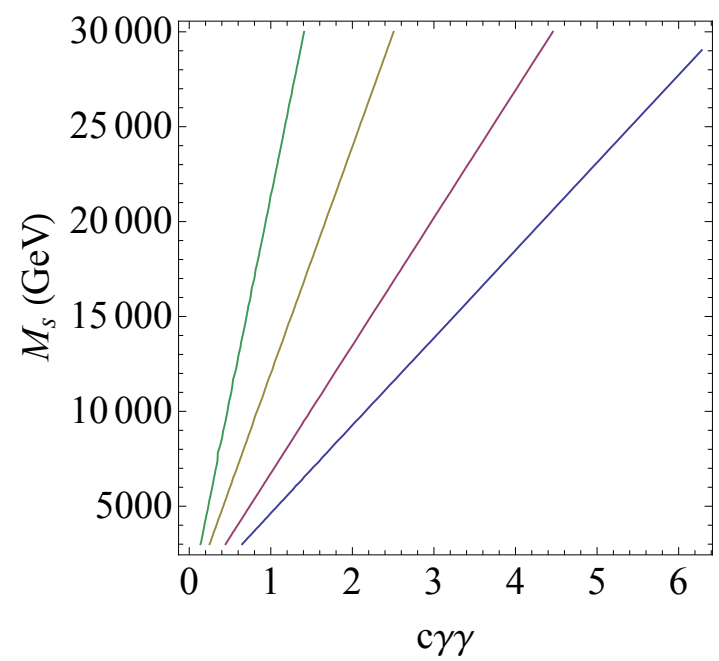

Figure 7. Best fit contours of diphoton cross section $\sigma_{\mathrm{LHC13}} \sim 5 \mathrm{fb}$ produced via photon fusion $\gamma \gamma \rightarrow \varphi \rightarrow \gamma \gamma$. The four lines (blue, red, yellow, green) are for $\Gamma_{\text {total }}=45,10,1.0,0.1 \mathrm{GeV}$, respectively.

where

$$
\mathcal{B}(\varphi \rightarrow \gamma \gamma)=\frac{2.3 \times 10^{6} c_{\gamma \gamma}^{2}}{\pi}\left(\frac{M_{s}}{\mathrm{GeV}}\right)^{-2}\left(\frac{\Gamma_{\text {total }}}{45 \mathrm{GeV}}\right)^{-1} .
$$

Substituting (8) into (7), and demanding (7) reproduces the diphoton signal (3) we obtain an equation connecting $c_{\gamma \gamma}$ with $M_{s}$ for given $\Gamma_{\text {total }}$. In Fig. 7 we show the best fit contours for $\sigma_{\mathrm{LHC13}} \sim 5 \mathrm{fb}$ and total widths $\Gamma_{\text {total }}=45,10,1,0.1 \mathrm{GeV}$. We assume that for a broad resonance the missing fraction of the decay width arises from the coupling of $\varphi$ to some fermion bulk fields. These hidden fermions could make a contribution to the dark matter content of the universe [55]. We conclude that for both the narrow and the broad resonance hypotheses there is an allowed region of the parameter space which is consistent with the experimental lower bound of $M_{s} \simeq 7 \mathrm{TeV}$ [49] and reproduces the LHC13 signal. For the broad resonance hypothesis, $7 \lesssim M_{s} / \mathrm{TeV} \lesssim 30$.

The total photo-production cross section at LHC8 is [50]

$$
\sigma_{\mathrm{LHC} 8}(\gamma \gamma \rightarrow \varphi \rightarrow \gamma \gamma)=1.4 \mathrm{pb}\left(\frac{\Gamma_{\text {total }}}{45 \mathrm{GeV}}\right) \mathcal{B}^{2}(\varphi \rightarrow \gamma \gamma),
$$

showing consistency with the 95\% CL upper limit [21].

The assumed coupling of $\varphi$ to the hypercharge field strength yields additional decay channels in the visible sector, namely $\varphi \rightarrow \gamma Z$ and $\varphi \rightarrow Z Z$, with

$$
\frac{\Gamma_{\gamma Z}}{\Gamma_{\gamma \gamma}}=2 \tan ^{2} \theta_{W} \approx 0.6 \text { and } \frac{\Gamma_{Z Z}}{\Gamma_{\gamma \gamma}}=\tan ^{4} \theta_{W} \approx 0.08 .
$$

This prediction is in agreement with the recent upper limit reported in by the ATLAS Collaboration from searches in the $\gamma Z$ channel [56].

We now turn to discuss the production via gluon fusion. We parametrize the coupling of $\varphi$ to the gluon by the following vertex

$$
\frac{c_{g g}}{M_{s}} \varphi G^{2} .
$$




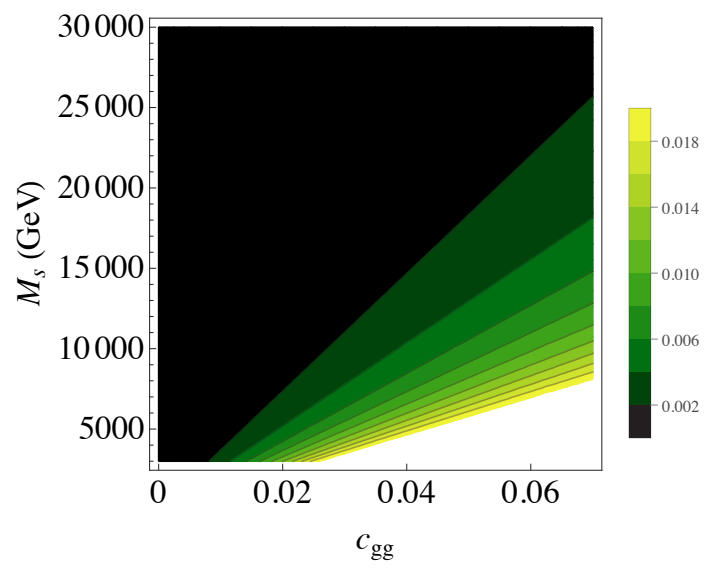

Figure 8. Contours of constant partial width $\Gamma_{g g}$. The color encoded scales are in $\mathrm{GeV}$.

The partial decay width of $\varphi$ to dijets is

$$
\Gamma_{g g}=8 \frac{c_{g g}^{2}}{4 \pi} \frac{M_{\varphi}^{3}}{M_{s}^{2}}
$$

In Fig. 8 we show a scan of the parameter space $\left(c_{g g}, M_{s}\right)$ for constant values of $\Gamma_{g g}$ as obtained from (12).

In the narrow width approximation the cross section for diphoton production via gluon fusion is given by [53]

$$
\sigma_{\mathrm{LHCl} 3}(g g \rightarrow \varphi \rightarrow \gamma \gamma)=5.8 \times 10^{3} \mathrm{pb} c_{g g}^{2}\left(\frac{M_{s}}{\mathrm{TeV}}\right)^{-2} \mathcal{B}(\varphi \rightarrow \gamma \gamma)
$$

and

$$
\sigma_{\mathrm{LHC} 8}(g g \rightarrow \varphi \rightarrow \gamma \gamma)=1.2 \times 10^{3} \mathrm{pb} c_{g g}^{2}\left(\frac{M_{s}}{\mathrm{TeV}}\right)^{-2} \mathcal{B}(\varphi \rightarrow \gamma \gamma)
$$

Substituting (3) and (8) into (13) we arrive at the targeting constraint equation connecting $c_{g g}, c_{\gamma \gamma}$, and $M_{s}$, for given $\Gamma_{\text {total }}$.

As of today the upper limit on dijet production at $M_{j j}=750 \mathrm{GeV}$ is dominated by LHC8 data, $\sigma_{\mathrm{LHC} 8}(p p \rightarrow j j)<2.5 \mathrm{pb}$ at $95 \% \mathrm{CL}$ [57]. The cross section for dijet production is

$$
\sigma_{\mathrm{LHC} 8}(g g \rightarrow \varphi \rightarrow g g)=7.6 \times 10^{3} \mathrm{pb}_{g g}^{4}\left(\frac{M_{s}}{\mathrm{TeV}}\right)^{-4}\left(\frac{\Gamma_{\text {total }}}{45 \mathrm{GeV}}\right) .
$$

Imposing the dijet constraint, $\sigma_{\mathrm{LHC} 8}(p p \rightarrow j j)<2.5 \mathrm{pb}$, on (15) we obtain the excluded region of the $\left(c_{g g}, c_{\gamma \gamma}\right)$ plane. The allowed region of the $\left(c_{g g}, c_{\gamma \gamma}\right)$ parameter space, which explains the observed diphoton excess at LHC13 and is consistent with LHC8 data, is shown in Fig. 9 for illustrative values of the string scale, $M_{s}=7 \mathrm{TeV}$ and $12 \mathrm{TeV}$. From (13) and (14) the LHC13/LHC8 luminosity ratio is found to be

$$
\frac{\mathcal{L}_{g g}(\sqrt{s}=13 \mathrm{TeV})}{\mathcal{L}_{g g}(\sqrt{s}=8 \mathrm{TeV})}=4.7 .
$$

Therefore, the production of $\varphi$ by gluon fusion is consistent with the lack of a diphoton excess in LHC8 data. As we have seen, imposing the LHC8 dijet limit [57] further constrains the $\left(c_{g g}, c_{\gamma \gamma}\right)$ 

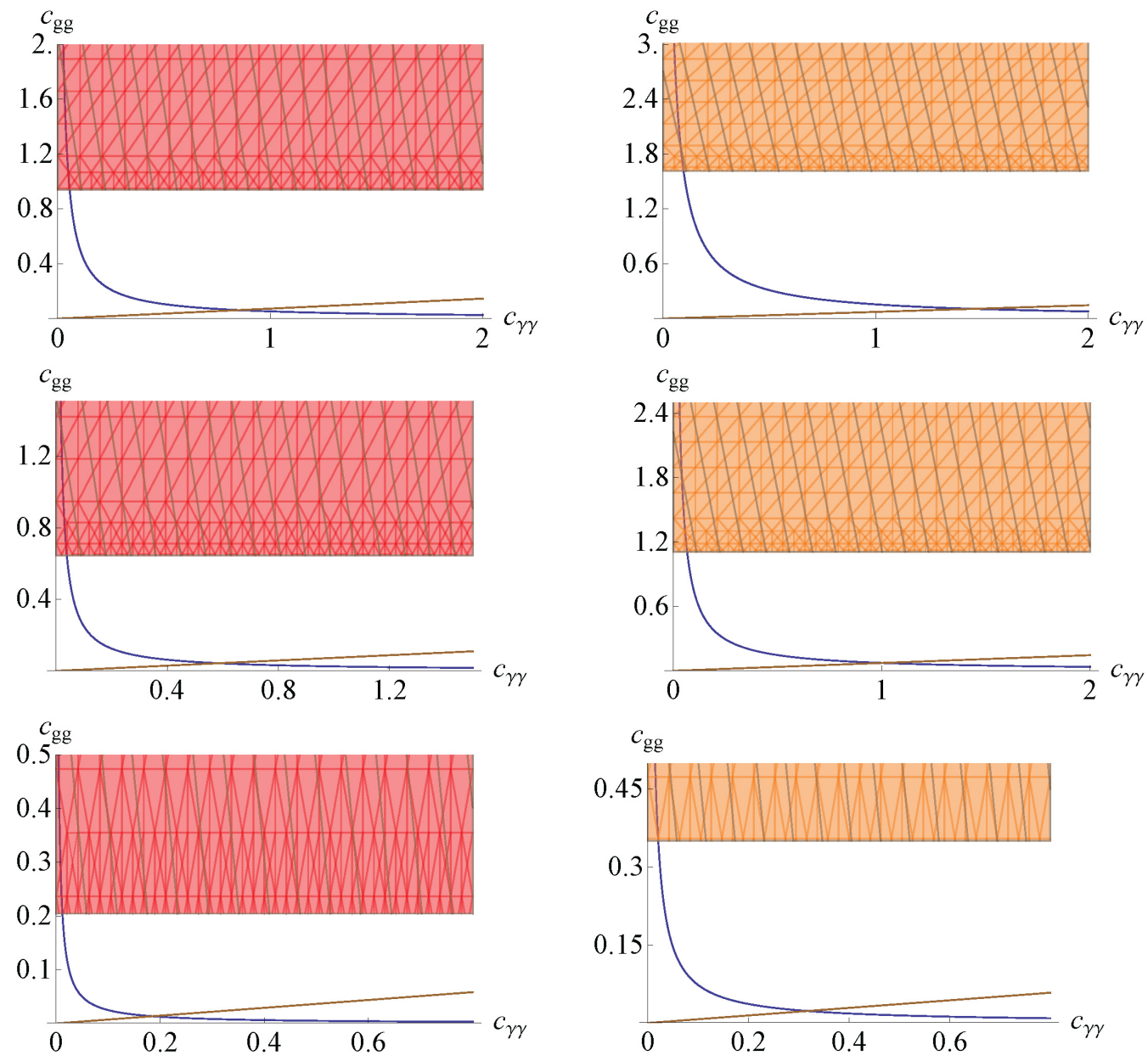

Figure 9. Allowed parameter space on the $c_{g g}$ vs $c_{\gamma \gamma}$ plane for $M_{s}=7 \mathrm{TeV}$ (left) and $M_{s}=12 \mathrm{TeV}$. The blue curves indicate the best fit of diphoton cross section $\left(\sigma_{\mathrm{LHC} 13}=5 \mathrm{fb}\right)$ for $M_{s}=7 \mathrm{TeV}$ (left) and $M_{s}=12 \mathrm{TeV}$ (right). The (red and orange) shaded regions are excluded at 95\% CL by null results on dijet searches. The slanted (brown) curve determines the transition between $c_{g g}$ and $c_{\gamma \gamma}$ dominance at production. Gluon fusion dominates above this curve. Results for three possible total widths are exhibited in the vertical columns, $\Gamma_{\text {total }}=$ 45, 10, $0.1 \mathrm{GeV}$, downwards.

parameter space. However, it remains the case that for $7 \lesssim M_{s} / \mathrm{TeV} \lesssim 30$ there is always some allowed region of the $\left(c_{g g}, c_{\gamma \gamma}\right)$ plane.

\section{Effective Planck mass and the inflation scale}

Low scale gravity with large extra dimensions is actually a particular case of a more general framework, where the UV cutoff is lower than the Planck scale due to the existence of a large number of particle species coupled to gravity [58]. Indeed, it was shown that the effective UV cutoff $M_{U V}$ is 
given by

$$
M_{U V}^{2}=M_{P}^{2} / N
$$

where the counting of independent species $N$ takes into account all particles which are not broad resonances, having a width less than their mass. For instance, in braneworld gravity with $n$ large extra dimensions of average size $R$, the particle species are the Kaluza-Klein (KK) excitations of the graviton (and other possible bulk modes), whose number at a given energy scale $E_{*}$ is given by

$$
N \simeq R^{n} E_{*}^{n}
$$

Here, we work out the consequences of this scale dependence of the strength of gravity for inferring various quantities during inflation [2], which we take to be driven by a single field for economy of discussion and because the data doesn't compel us to consider otherwise [59]. As is to be expected, all dimensionless observables such as the amplitude and spectral properties of the perturbations are unaffected by the changing strength of gravity at inflationary energies. However, when one tries to infer an absolute energy scale for inflation, one finds that it is undetermined commensurate with (17) up to the unknown spectrum of universally coupled species between laboratory scales and the inflationary scale, the details of which we elaborate upon in the following.

According to the inflationary paradigm, the primordial perturbations observed in the CMB were created at horizon crossing during the quasi de Sitter (dS) phase of early accelerated expansion sourced by the inflaton field. Therefore all quantities that enter calculations of primordial correlation functions (which we subsequently relate to observables in the $\mathrm{CMB}$ ) refer to quantities at the scale at which inflation occurred. We denote all quantities measured at the scale of inflation with a starred subscript. The dominant contribution to the temperature anisotropies comes from adiabatic perturbations ${ }^{2}$ sourced by the comoving curvature perturbation $\mathcal{R}$, defined as the conformal factor of the 3-metric $h_{i j}$ in comoving gauge:

$$
h_{i j}(t, x)=a^{2}(t) e^{2 \mathcal{R}(t, x)} \hat{h}_{i j} ; \quad \hat{h}_{i j}:=\exp \left[\gamma_{i j}\right]
$$

with $\partial_{i} \gamma_{i j}=\gamma_{i i}=0$ defining transverse traceless graviton perturbations. The temperature anisotropies are characterized by the dimensionless power spectrum for $\mathcal{R}$, whose amplitude is given by

$$
\mathcal{P}_{\mathcal{R}}:=\frac{H_{*}^{2}}{8 \pi^{2} M_{*}^{2} \epsilon_{*}}=\mathcal{A} \times 10^{-10},
$$

where $\epsilon_{*}:=-\dot{H}_{*} / H_{*}^{2}, H_{*}$ being the Hubble factor during inflation. Given that $\mathcal{R}$ is conserved on superhorizon scales (in the absence of entropy perturbations), this immediately relates to the amplitude of the late time $\mathrm{CMB}$ anisotropies, which fixes $\mathcal{A} \sim 22.15$ [59]. The tensor anisotropies are characterized by the tensor power spectrum

$$
\mathcal{P}_{\gamma}:=2 \frac{H_{*}^{2}}{\pi^{2} M_{*}^{2}},
$$

Taking the ratio of the above with (20), we find the tensor to scalar ratio

$$
r_{*}:=\frac{\mathcal{P}_{\gamma}}{\mathcal{P}_{\mathcal{R}}}=16 \epsilon_{*}
$$

\footnotetext{
${ }^{2}$ In what follows, we assume that all of the extra species have sufficiently suppressed couplings to the inflaton during inflation (e.g. either through derivative couplings or as Planck suppressed interactions) so that isocurvature perturbations are not significantly generated. This is trivially true for hidden sector fields.
} 
Therefore any determination of $r_{*}$, either through direct measurements of the stochastic background of primordial gravitational waves or through their secondary effects on the polarization of the CMB [60-62] allows us in principle to fix the scale of inflation:

$$
H_{*}=M_{*}\left(\frac{\pi^{2} \mathcal{A} r_{*}}{2 \cdot 10^{10}}\right)^{1 / 2}:=\Upsilon=1.05 \sqrt{r}_{*} \times 10^{-4} .
$$

We see that any measurements of $r_{*}$ determines the scale of inflation up to our ignorance of the effective strength of gravity at the scale $H_{*}$, given by $M_{*} \sim \frac{M_{P}}{\sqrt{N}}$, where $N$ is the effective number of all universally coupled species up to the scale $H_{*}$ - whether they exist in the visible sector or in any hidden sector. Note that as one lowers the scale of strong gravity, the maximum reheating temperature $T_{i}$ is necessarily lowered as well, since it cannot be higher than the inflation scale. Conservatively, $T_{i}$ cannot be too far below the TeV scale without spoiling the standard scenarios of big bang cosmologyin particular, mechanisms for Leptogenesis and Baryogenesis which can occur no lower than the electroweak scale [63].

An example of extra species is KK graviton modes in theories of low scale gravity and large extra dimensions. In this case $N_{*}=N_{\mathrm{TT}} / 2$, where $N_{\mathrm{TT}}$ counts the two polarizations of the massless graviton plus the number of contributing TT Kaluza-Klein (KK) resonances with masses below the momentum transfer in question. On a Minkowski background for example, in the presence of large (but stabilized) extra dimensions, this is nothing more than the usual manner in which extra dimensions open up ${ }^{3}$. An important qualification to the discussion initiated in [2] was raised by the authors of [64], who pointed out that on a dS background $N_{\mathrm{TT}}$ could not receive any contributions from KK gravitons since the former must respect the Higuchi bound [65] whereby massive gravitons are gapped as $m_{\mathrm{KK}}^{2}>2 H^{2}$. Therefore, it was reasoned that KK gravitons could not enhance any processes involving graviton exchange at inflationary energies. However, even in this very special limit (where an enhanced symmetry results in important qualitative differences compared to quasi dS backgrounds), KK gravitons with masses $m_{\mathrm{KK}}^{2} \gg 2 H^{2}$ can still contribute non-trivially to the observed tensor power. This occurs without modifications to the spectral properties of the curvature perturbations, rendering the inferred energy scale of inflation effectively uncertain from any potential observation of primordial B-modes. This is because of the well known fact that KK modes of the graviton can couple much more strongly to 4-dimensional (4D) localized sources than the zero mode in the presence of warped extra dimensions [66]. Indeed, in an example studied in [67], one finds that individual KK modes of the graviton couple with the characteristic effective strength

$$
M_{*} \sim M_{\mathrm{pl}} e^{-3 z_{c} / 2} \ll M_{\mathrm{pl}}
$$

whenever $z_{c} \gg 1$, where $e^{z_{c}}$ parametrizes the warping. If this were the context in which inflation actually occurs, attempts to infer an absolute scale for inflation from any putative observation of primordial B-modes in the CMB at a fixed scale would be rendered uncertain up to the factor

$$
N_{\mathrm{TT}}^{\mathrm{eff}}=2\left(1+e^{3\left(z_{c}-\mathcal{N}_{\checkmark}\right)}\right)
$$

where $\mathcal{N}_{\sqrt{ }}$ is the number of e-folds since horizon exit of the pivot scale at which we fix the tensor amplitude.

\footnotetext{
${ }^{3}$ We remind the reader that the moduli mass that stabilizes any extra dimensions is independent of the expectation value of the moduli (i.e. its size). It is the latter that determines the mass splitting of the KK excitations.
} 


\section{References}

[1] I. Antoniadis, N. Arkani-Hamed, S. Dimopoulos and G. R. Dvali, Phys. Lett. B 436 (1998) 257 [arXiv:hep-ph/9804398]

[2] I. Antoniadis and S. P. Patil, Eur. Phys. J. C75 (2015) 182 [arXiv:1410.8845 [hep-th]].

[3] C. Angelantonj and A. Sagnotti, Phys. Rept. 371 (2002) 1 [Erratum-ibid. 376 (2003) 339] [arXiv:hep-th/0204089].

[4] I. Antoniadis, Phys. Lett. B 246 (1990) 377.

[5] D. J. Kapner, T. S. Cook, E. G. Adelberger, J. H. Gundlach, B. R. Heckel, C. D. Hoyle and H. E. Swanson, Phys. Rev. Lett. 98 (2007) 021101.

[6] J. C. Long and J. C. Price, Comptes Rendus Physique 4 (2003) 337; R. S. Decca, D. Lopez, H. B. Chan, E. Fischbach, D. E. Krause and C. R. Jamell, Phys. Rev. Lett. 94 (2005) 240401; R. S. Decca et al., arXiv:0706.3283 [hep-ph]; S. J. Smullin, A. A. Geraci, D. M. Weld, J. Chiaverini, S. Holmes and A. Kapitulnik, arXiv:hep-ph/0508204; H. Abele, S. Haeßler and A. Westphal, in 271th WE-Heraeus-Seminar, Bad Honnef (2002).

[7] I. Antoniadis and K. Benakli, Phys. Lett. B 326 (1994) 69.

[8] I. Antoniadis, K. Benakli and M. Quirós, Phys. Lett. B 331 (1994) 313 and Phys. Lett. B 460 (1999) 176; P. Nath, Y. Yamada and M. Yamaguchi, Phys. Lett. B 466 (1999) 100 T. G. Rizzo and J. D. Wells, Phys. Rev. D 61 (2000) 016007; T. G. Rizzo, Phys. Rev. D 61 (2000) 055005; A. De Rujula, A. Donini, M. B. Gavela and S. Rigolin, Phys. Lett. B 482 (2000) 195.

[9] G.F. Giudice, R. Rattazzi and J.D. Wells, Nucl. Phys. B 544 (1999) 3; E.A. Mirabelli, M. Perelstein and M.E. Peskin, Phys. Rev. Lett. 82 (1999) 2236; T. Han, J.D. Lykken and R. Zhang, Phys. Rev. D 59 (1999) 105006; K. Cheung, W.-Y. Keung, Phys. Rev. D 60 (1999) 112003; C. Balázs et al., Phys. Rev. Lett. 83 (1999) 2112; J.L. Hewett, Phys. Rev. Lett. 82 (1999) 4765.

[10] L.A. Anchordoqui, H. Goldberg, D. Lust, S. Nawata, S. Stieberger and T.R. Taylor, Phys. Rev. Lett. 101 (2008) 241803 [arXiv:0808.0497 [hep-ph]].

[11] L. A. Anchordoqui, I. Antoniadis, D. C. Dai, W. Z. Feng, H. Goldberg, X. Huang, D. Lust and D. Stojkovic et al., Phys. Rev. D 90 (2014) 6, 066013 [arXiv:1407.8120 [hep-ph]].

[12] I. Antoniadis, E. Kiritsis and T. N. Tomaras, Phys. Lett. B 486, 186 (2000) [arXiv:hepph/0004214]; I. Antoniadis, E. Kiritsis, J. Rizos and T. N. Tomaras, Nucl. Phys. B 660, 81 (2003) [arXiv:hep-th/0210263].

[13] G. Shiu and S.-H. H. Tye, Phys. Rev. D 58 (1998) 106007; Z. Kakushadze and S.-H. H. Tye, Nucl. Phys. B 548 (1999) 180; L. E. Ibáñez, C. Muñoz and S. Rigolin, Nucl. Phys. B 553 (1999) 43.

[14] I. Antoniadis, E. Kiritsis and J. Rizos, Nucl. Phys. B 637, 92 (2002) [arXiv:hep-th/0204153];

[15] L. A. Anchordoqui, I. Antoniadis, H. Goldberg, X. Huang, D. Lüst and T. R. Taylor, Phys. Rev. D 85, 086003 (2012) [arXiv:1107.4309 [hep-ph]].

[16] G.T. Horowitz and J. Polchinski, Phys. Rev. D 55 (1997) 6189.

[17] ATLAS Collaboration, ATLAS-CONF-2015-081; M. Delmastro [on behalf of the ATLAS Collaboration], 51st Rencontres de Moriond (Electroweak session) 17 May 2016, La Thuile (Italy).

[18] CMS Collaboration, CMS-PAS-EXO-15-004; P. Musella [on behalf of the CMS Collaboration], 51st Rencontres de Moriond (Electroweak session) 17 May 2016, La Thuile (Italy); CMS Collaboration, CMS-PAS-EXO-16-018.

[19] R. Franceschini et al., JHEP 1603 (2016) 144 [arXiv:1512.04933 [hep-ph]].

[20] G. Aad et al. [ATLAS Collaboration], Phys. Rev. D 92, 032004 (2015) [arXiv:1504.05511 [hepex]]. 
[21] V. Khachatryan et al. [CMS Collaboration], Phys. Lett. B 750, 494 (2015) [arXiv:1506.02301 [hep-ex]].

[22] L. A. Anchordoqui, I. Antoniadis, H. Goldberg, X. Huang, D. Lüst and T. R. Taylor, Phys. Lett. B 755, 312 (2016) [arXiv:1512.08502 [hep-ph]]; arXiv:1603.08294 [hep-ph].

[23] R. Blumenhagen, M. Cvetic, P. Langacker and G. Shiu, Ann. Rev. Nucl. Part. Sci. 55, 71 (2005) [hep-th/0502005].

[24] R. Blumenhagen, B. Kors, D. Lüst and S. Stieberger, Phys. Rept. 445, 1 (2007) [hepth/0610327].

[25] D. Cremades, L. E. Ibanez and F. Marchesano, JHEP 0307, 038 (2003) [hep-th/0302105].

[26] L. A. Anchordoqui, I. Antoniadis, H. Goldberg, X. Huang, D. Lüst and T. R. Taylor, Phys. Lett. B 749, 484 (2015) [arXiv:1507.05299 [hep-ph]].

[27] M. B. Green and J. H. Schwarz, Phys. Lett. B 149, 117 (1984).

[28] E. Witten, Phys. Lett. B 149, 351 (1984).

[29] M. Dine, N. Seiberg, X. G. Wen and E. Witten, Nucl. Phys. B 289, 319 (1987).

[30] W. Lerche, B. E. W. Nilsson, A. N. Schellekens and N. P. Warner, Nucl. Phys. B 299, 91 (1988).

[31] L. E. Ibanez and F. Quevedo, JHEP 9910, 001 (1999) [hep-ph/9908305].

[32] D. M. Ghilencea, L. E. Ibanez, N. Irges and F. Quevedo, JHEP 0208, 016 (2002) [hep$\mathrm{ph} / 0205083]$.

[33] J. J. Heckman, Nucl. Phys. B 906 (2016) 231 [arXiv:1512.06773 [hep-ph]].

[34] M. Cvetic, J. Halverson and P. Langacker, arXiv:1512.07622 [hep-ph].

[35] L. E. Ibanez and V. Martin-Lozano, arXiv:1512.08777 [hep-ph].

[36] E. Palti, Nucl. Phys. B 907 (2016) 597 [arXiv:1601.00285 [hep-ph]].

[37] A. Karozas, S. F. King, G. K. Leontaris and A. K. Meadowcroft, Phys. Lett. B 757 (2016) 73 [arXiv:1601.00640 [hep-ph]].

[38] A. E. Faraggi and J. Rizos, Eur. Phys. J. C 76 (2016) no.3, 170 [arXiv:1601.03604 [hep-ph]].

[39] P. Anastasopoulos and M. Bianchi, arXiv:1601.07584 [hep-th].

[40] M. Cvetic, J. Halverson and P. Langacker, arXiv:1602.06257 [hep-ph].

[41] T. Li, J. A. Maxin, V. E. Mayes and D. V. Nanopoulos, arXiv:1602.09099 [hep-ph].

[42] G. K. Leontaris and Q. Shafi, arXiv:1603.06962 [hep-ph].

[43] S. Abel and V. V. Khoze, JHEP 1605 (2016) 063 [arXiv:1601.07167 [hep-ph]].

[44] T. Higaki, K. S. Jeong, N. Kitajima and F. Takahashi, Phys. Lett. B 755, 13 (2016) [arXiv:1512.05295 [hep-ph]].

[45] E. Megias, O. Pujolas and M. Quiros, JHEP 1605 (2016) 137 [arXiv:1512.06106 [hep-ph]].

[46] I. Ben-Dayan and R. Brustein, arXiv:1601.07564 [hep-ph].

[47] N. D. Barrie, A. Kobakhidze, M. Talia and L. Wu, Phys. Lett. B 755, 343 (2016) [arXiv:1602.00475 [hep-ph]].

[48] L. Aparicio, A. Azatov, E. Hardy and A. Romanino, JHEP 1605 (2016) 077 [arXiv:1602.00949 [hep-ph]].

[49] V. Khachatryan et al. [CMS Collaboration], Phys. Rev. Lett. 116 (2016) no.7, 071801 [arXiv:1512.01224 [hep-ex]].

[50] L. A. Harland-Lang, V. A. Khoze and M. G. Ryskin, JHEP 1603 (2016) 182 [arXiv:1601.07187 [hep-ph]].

[51] ATLAS Collaboration, Technical Report CERN- LHCC-2011-012. LHCC-I-020, CERN, Geneva, Nov. 2011. 
[52] M Albrow et al. [CMS and TOTEM Collaborations], Technical Report CERN-LHCC-2014-021. TOTEM-TDR-003. CMS-TDR-13, CERN, Geneva, Sep. 2014.

[53] C. Csaki, J. Hubisz, S. Lombardo and J. Terning, arXiv:1601.00638 [hep-ph].

[54] A. D. Martin and M. G. Ryskin, J. Phys. G 43, no. 4, 04LT02 (2016) [arXiv:1601.07774 [hep$\mathrm{ph}]$.

[55] K. R. Dienes and B. Thomas, Phys. Rev. D 85, 083523 (2012) [arXiv:1106.4546 [hep-ph]]; Phys. Rev. D 85, 083524 (2012) [arXiv:1107.0721 [hep-ph]].

[56] ATLAS Collaboration, ATLAS-CONF-2016-010.

[57] G. Aad et al. [ATLAS Collaboration], Phys. Rev. D 91, 052007 (2015) [arXiv:1407.1376 [hepex]].

[58] G. Dvali, arXiv:0706.2050 [hep-th]; Int. J. Mod. Phys. A 25 (2010) 602 [arXiv:0806.3801 [hep-th]]; G. Dvali and M. Redi, Phys. Rev. D 77 (2008) 045027 [arXiv:0710.4344 [hep-th]]; R. Brustein, G. Dvali and G. Veneziano, JHEP 0910 (2009) 085 [arXiv:0907.5516 [hep-th]]; G. Dvali and C. Gomez, Phys. Lett. B 674 (2009) 303.

[59] P. A. R. Ade et al. [Planck Collaboration], Astron. Astrophys. (2014) [arXiv:1303.5076 [astroph.CO]]; arXiv:1303.5082 [astro-ph.CO].

[60] A. G. Polnarev, Sov. Astron. 29, 607 (1985).

[61] M. Kamionkowski, A. Kosowsky and A. Stebbins, Phys. Rev. Lett. 78, 2058 (1997) [astro$\mathrm{ph} / 9609132]$.

[62] U. Seljak and M. Zaldarriaga, Phys. Rev. Lett. 78, 2054 (1997) [astro-ph/9609169].

[63] M. Trodden, Rev. Mod. Phys. 71, 1463 (1999) [hep-ph/9803479]; S. Davidson, E. Nardi and Y. Nir, Phys. Rept. 466, 105 (2008) [arXiv:0802.2962 [hep-ph]].

[64] M. Kleban, M. Mirbabayi and M. Porrati, JCAP 1601, no. 01, 017 (2016) [arXiv:1508.01527 [hep-th]].

[65] A. Higuchi, Nucl. Phys. B 282, 397 (1987).

[66] L. Randall and R. Sundrum, Phys. Rev. Lett. 83, 3370 (1999) [hep-ph/9905221].

[67] I. Antoniadis and S. P. Patil, in preparation. 